\title{
Current Practices for Outpatient Initiation of Levodopa-Carbidopa Intestinal Gel for Management of Advanced Parkinson's Disease in the United States
}

\author{
Fahd Amjad · Danish Bhatti · Thomas L. Davis · Odinachi Oguh • \\ Rajesh Pahwa $\cdot$ Pavnit Kukreja $\cdot$ Jorge Zamudio $\cdot$ Leonard Verhagen Metman
}

Received: March 27, 2019 / Published online: July 5, 2019

(C) The Author(s) 2019
Enhanced Digital Features To view enhanced digital features for this article go to https://doi.org/10.6084/ m9.figshare.8248058.

F. Amjad ( $\square)$

Department of Neurology, Georgetown University Hospital Pasquerilla Healthcare Center, 7th Floor, 3800 Reservoir Road NW, Washington, DC 20007, USA

e-mail: Fahdvr@hotmail.com

D. Bhatti

Department of Neurological Sciences, University of Nebraska Medical Center, 988440 Nebraska Medical Center, Omaha, NE 68198, USA

T. L. Davis

Department of Neurology, Vanderbilt University Medical Center, 1301 Medical Center Drive \#3930, Nashville, TN 37212, USA

O. Oguh

Department of Neurology, University of Florida College of Medicine-Jacksonville, 9th Floor, Tower I, 580 West 8th Street, Jacksonville, FL 32209, USA levodopa. First, levodopa is delivered in a continuous rather than intermittent, pulsatile fashion. Second, delivery to levodopa's site of absorption in the jejunum bypasses the stomach, thereby avoiding issues with erratic gastric emptying. In blinded prospective clinical trials and observational studies, LCIG has been shown to significantly decrease "off" time, increase "on" time without troublesome dyskinesia, and reduce dyskinesia. Consistent with procedures in previous studies, LCIG initiation and titration in the pivotal US clinical trial were performed in the inpatient setting and followed a standardized protocol. In clinical practice, however, initiation and titration of LCIG have a

\section{R. Pahwa}

Department of Neurology, University of Kansas Medical Center, 3599 Rainbow Blvd, Kansas City, KS 66103, USA

\section{P. Kukreja · J. Zamudio}

AbbVie, Inc, 1 North Waukegan Road, North Chicago, IL 60064, USA

\section{V. Metman}

Department of Neurological Sciences, Rush University, 1725 W Harrison Street, Suite 755, Chicago, IL 60612, USA 
great degree of flexibility and, in the US, almost always take place in the outpatient setting. Nonetheless, there remains a significant amount of clinician uncertainty regarding titration in outpatient clinical practice. This review aims to shed light on and provide guidance as to the current methods of titration in the outpatient setting, as informed by the medical literature and the authors' experiences.

Funding: AbbVie, Inc.

Plain Language Summary: Plain language summary available for this article.

\section{PLAIN LANGUAGE SUMMARY}

Results from recent studies have shown that continuous infusion of levodopa-carbidopa intestinal gel (LCIG) into the jejunum (a part of the small intestine) effectively manages the motor and nonmotor complications (e.g., tremor, extreme stiffness in arms and legs, difficulty walking, and impaired balance) experienced by patients with advanced Parkinson's disease (PD). LCIG is administered by a portable pump directly into the patient's jejunum by a permanent tube that is inserted surgically. LCIG therapy is beneficial to advanced PD patients over orally administered carbidopa/ levodopa for two reasons. First, oral carbidopa/ levodopa moves from the stomach to the small intestine where it is intermittently absorbed into the blood stream. LCIG is administered continuously and offers better symptom control for longer. Results from clinical trials and observational studies have shown that LCIG significantly decreases "off" time (poor motor control) and increases "on" time (good motor control) in advanced PD patients without troublesome dyskinesia, which results from the higher doses of oral levodopa required to treat the symptoms. Second, LCIG is absorbed in the jejunum, thereby bypassing the stomach where problems can occur because of inconsistent stomach emptying. In the US, titration of LCIG is performed mostly in an outpatient setting. Some clinicians may view titration of LCIG to be too complex and variable, so they avoid using LCIG therapy for their PD patients.
Fortunately, emerging data and clinicians' expanding experience with LCIG have shown that titration can be easily managed in an outpatient setting, allowing for more customized therapeutic regimens for patients.

Keywords: Carbidopa/levodopa enteral suspension; Continuous dopaminergic stimulation; Device-aided therapy; Duodopa; Duopa; LCIG; Neurology

\section{INTRODUCTION}

Levodopa-carbidopa intestinal gel (LCIG; known in the US as carbidopa-levodopa enteral suspension) is an aqueous carboxymethylcellulose gel suspension containing four parts levodopa to one part carbidopa monohydrate (4.63 $\mathrm{mg}$ carbidopa and $20 \mathrm{mg}$ levodopa per $\mathrm{ml})$, the same ratio as that provided in oral carbidopa-levodopa $25-100$ tablets. LCIG is administered via a portable pump directly to the proximal small intestine through a percutaneous endoscopic gastrojejunostomy (PEG-J) tube (Fig. 1) [1]. This system delivers medication to the site of levodopa absorption, thus avoiding issues associated with delayed or variable gastric emptying. Delivery of levodopa via infusion also permits finer adjustments in dose than are possible with oral administration. The mode of administration combined with continuous medication flow allows LCIG to provide more consistent circulating levels of levodopa and carbidopa compared with immediate-release oral carbidopa/levodopa treatment [2]. The goal of this approach is, hopefully, to generate sustained rather than intermittent stimulation of striatal dopaminergic neurons, thereby preventing the occurrence of "off" periods in patients with advanced Parkinson's disease (PD) [3].

LCIG (Duopa, AbbVie, Inc., North Chicago, IL, USA) was approved in the US in January 2015 for the treatment of motor fluctuations in patients with advanced PD, although it has been available in other parts of the world for $>$ 10 years (known as Duodopa ${ }^{\circledR}$ outside the US). LCIG has been shown to significantly reduce 


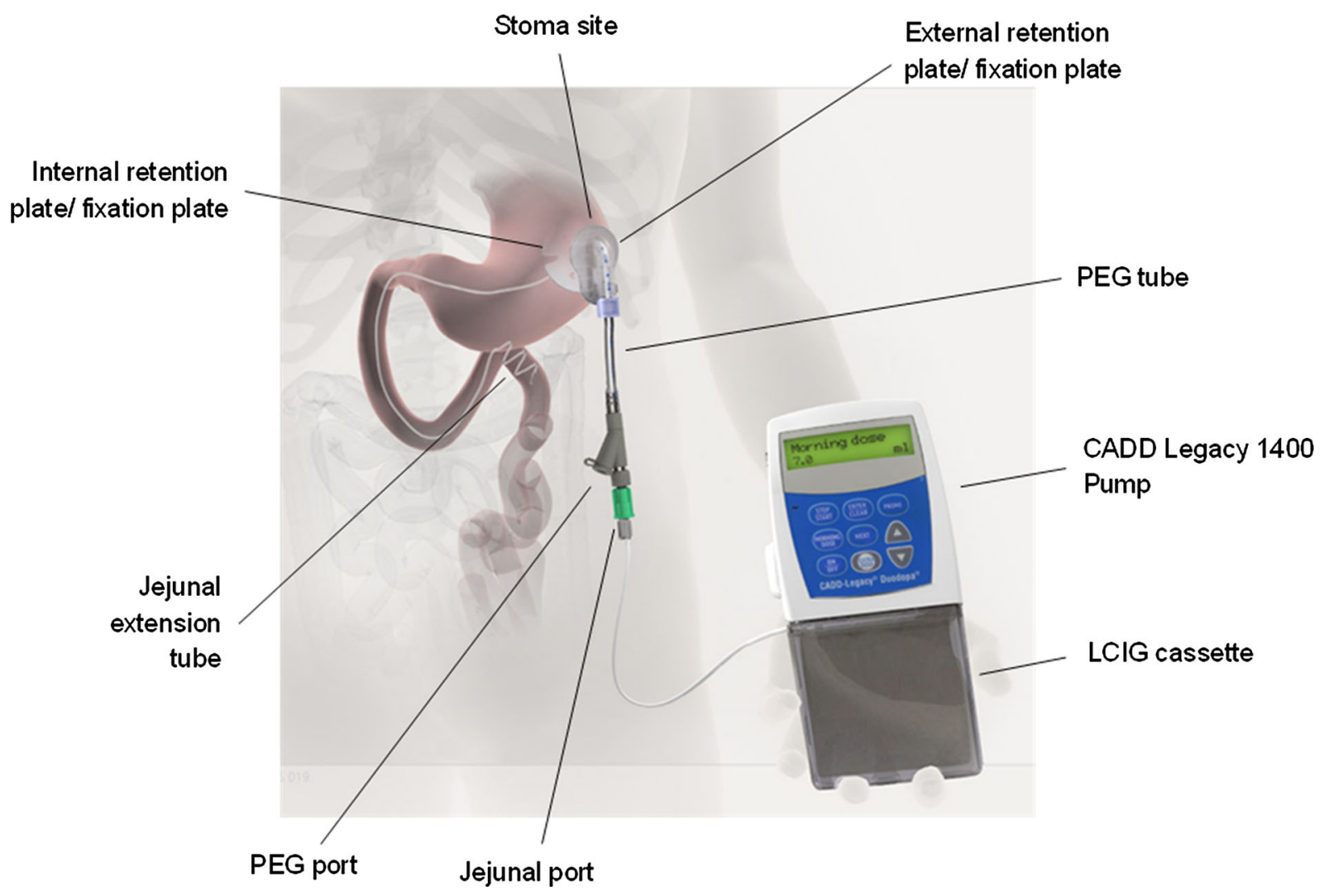

Fig. 1 The levodopa-carbidopa intestinal gel (LCIG) system. $P E G$ percutaneous endoscopic gastrojejunostomy. Image reproduced from Clin Transl Gastroenterol (Creative Commons license CC BY-NC-ND 4.0) [1]

"off" time and increase "on" time without troublesome dyskinesia in patients with advanced PD [4-11]. Marked improvements have also been observed in non-motor symptoms (e.g., sleep/fatigue, attention/memory, mood/cognition, gastrointestinal tract, urinary, sexual function) and in quality-of-life measures $[6-8,10-12]$. Benefits related to LCIG have proved to be durable [8-11], with reductions in mean daily "off" time maintained for as long as 9 years after initiating LCIG therapy [13].

The process of initiating and titrating LCIG has evolved with clinical studies and experience. When first introduced in Europe, a trial period of LCIG administration via a nasojejunal tube was standard to assess the patient's response prior to moving forward with PEG-J tube placement. By the advent of the first US trial, however, this was not considered a necessary introductory step; patients who met study-entry criteria proceeded to PEG-J tube placement without the intermediary test period
[6]. During the pivotal US trial, patients remained in the hospital for up to 2 weeks after PEG-J tube placement, at which time LCIG treatment was initiated and titration commenced [6]. This step has also been streamlined over time. In US clinical practice, LCIG initiation and titration are typically performed in the outpatient setting, an approach that is supported by findings in a recently published openlabel, phase $3 b$ study [10]. Clinical experience has yielded additional insights into the LCIG initiation and titration process, including identification of points in the process that can be optimized to meet patient and practice needs. In this review, we use clinical data and experience to guide clinicians through the steps involved in converting patients to LCIG therapy in the outpatient setting. This article is based on previously conducted studies and the authors' clinical experience and does not involve any new studies of human or animal subjects performed by any of the authors. 


\section{CANDIDATE DETERMINATION}

As noted above, LCIG therapy requires placement of a PEG-J tube. However, prior to PEG-J tube placement, patients are evaluated by their neurologist to determine if they are appropriate candidates for LCIG, as indicated by the presence of "off" periods despite optimized treatment with oral PD medications. The neurologist may also verify that the patient's most relevant symptoms are still levodopa responsive. During the pre-LCIG assessment, the patient management team will determine whether the patient can manage therapy on his or her own or, if a caregiver is needed, the support available is sufficient. In addition, the patient will need to be evaluated by the proceduralist (e.g., gastroenterologist, interventional radiologist or general surgeon) who will place the PEG-J tube to ensure the procedure will be feasible and the patient has no clinical or anatomic limitations that would interfere with tube placement.

A 2015 consensus statement [14] from an international panel of experts suggested that intrajejunal levodopa infusion is considered as both therapeutic and palliative in some countries for patients who have cognitive impairment or dementia. This group also suggested that LCIG may be considered for patients aged $>70$ years who have mild or moderate cognitive impairment or severe depression. Moreover, emerging clinical data support the safe use of LCIG in patients with PD and cognitive impairment [15]. In our clinical experience, the LCIG initiation and titration process does not differ among those individuals with cognitive impairment compared with individuals who have normal cognitive function.

\section{INITIAL TITRATION}

A schematic of the titration process from the PEG-J procedure through follow-up is presented in Fig. 2. The interval between placement of the PEG-J tube and initiation of LCIG is variable. In the outpatient titration study by Standaert and colleagues [10], the protocol mandated that LCIG was to be initiated within 5 days of the PEG-J procedure. Approximately two-thirds of patients commenced LCIG within 24 h of PEG-J tube placement, and 90\% had started LCIG within $48 \mathrm{~h}$ of the PEG-J procedure. On the other end of the spectrum, the outpatient model presented by Fasano et al. [16] recommends a minimum of 2 weeks between PEG-J tube placement and LCIG initiation "to allow the fistula track to establish", thereby potentially reducing the risk for site-related complications. Adverse events that occur more frequently during the early phases of LCIG treatment compared with the maintenance phase are often related to the procedure (e.g., procedural pain, stoma site irritation) [10]. Standard practice in the US involves scheduling patients for their first titration visit approximately 1 week after the PEG-J procedure to allow for adequate healing time, air absorption from the abdomen, and discontinuation of pain medications, which can affect treatment response. In rare cases such as when the patient lives a long distance from the clinic and has travel/logistic concerns, rapid initiation (i.e., within $24 \mathrm{~h}$ of PEG-J tube placement) may be considered.

The objective of the first visit is not to achieve the optimal dose, rather it is to achieve therapeutic benefit with further fine-tuning performed by the patient and clinician, either at home or in the office. Based on our clinical experience, during the first titration visit, patients will spend $1-6 \mathrm{~h}$ in the clinic, depending on the patient and clinician. A recent study reported similar findings [median time spent titrating LCIG was $3 \mathrm{~h}$ (range 0.5-5 h)] [17]. On arrival at the clinic, a brief motor examination is completed to provide a point of comparison for assessing response to therapy. Monitoring and pump programming are performed by clinic staff (e.g., nurse, nurse practitioner, physician assistant, or medical assistant), with periodic assessments made by the movement disorder specialist/neurologist.

\section{DETERMINING INITIAL LCIG DOSAGE}

The initial total daily dose of LCIG is calculated by adding the patient's total daytime oral 


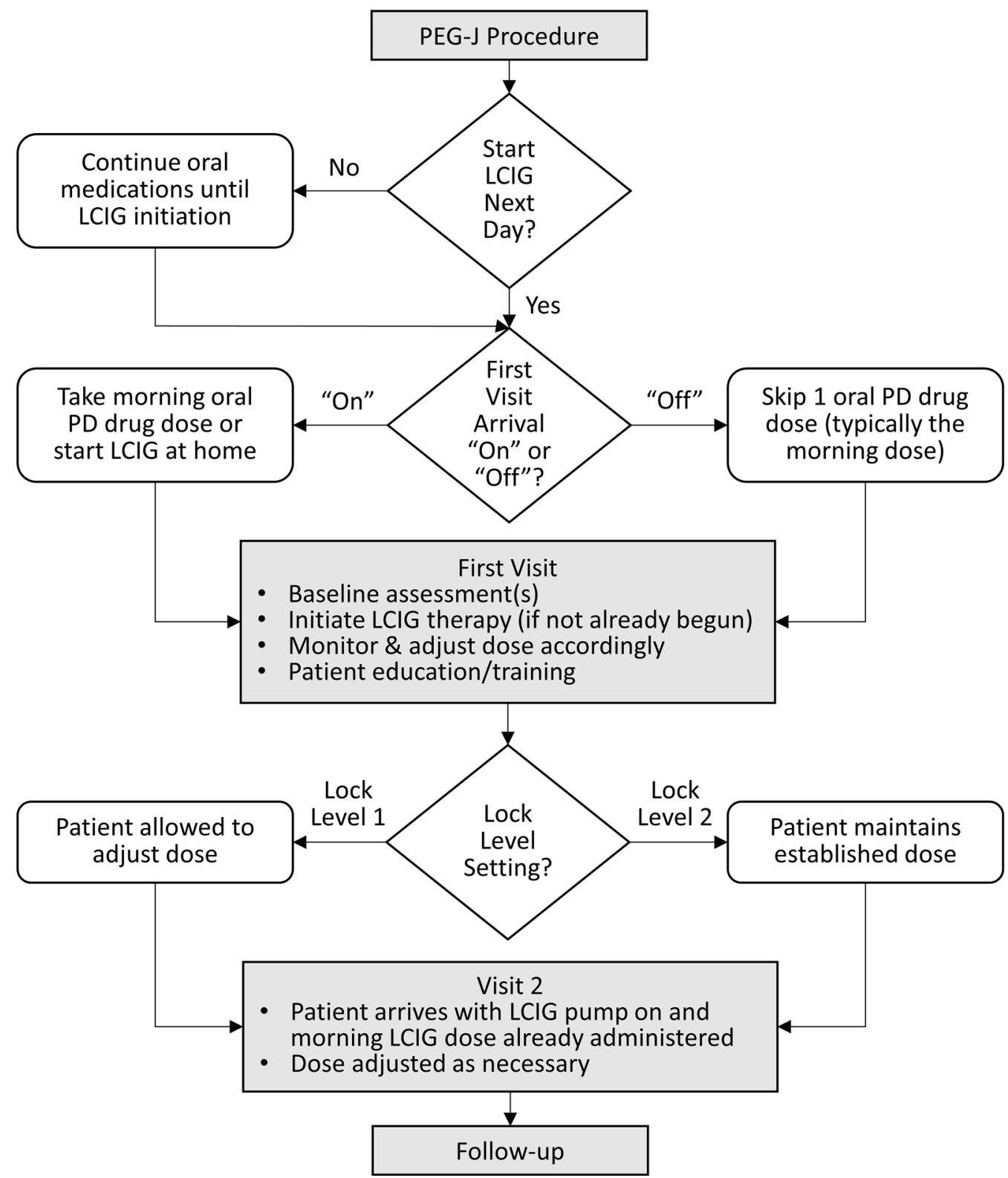

Fig. 2 Schematic of the levodopa-carbidopa intestinal gel (LCIG) titration process. Shaded rectangles indicate clinical touch points. Diamonds indicate decision points, including rapid (within $24 \mathrm{~h}$ ) versus delayed initiation of

levodopa dose and the levodopa equivalent dose of other PD medications (if these medications are to be discontinued at the time of transition to LCIG). The majority of clinicians do not discontinue other PD medications until the patient is stabilized on his or her levodopa dose to help mitigate unwanted side effects
LCIG after percutaneous endoscopic gastrojejunostomy (PEG-J) tube placement, arriving at the first visit in the "on" versus "off" states and Lock Level 1 versus Lock Level 2 programming. $P D$ Parkinson’s disease

such as dopamine agonist withdrawal syndrome [18], restless legs, increased akinesia and so forth. In most cases, the patient will receive LCIG treatment for $16 \mathrm{~h}$ per day and, if needed, oral levodopa for nighttime coverage. Treatment with oral levodopa during the day is replaced by the LCIG infusion. 
Although the goal of LCIG is not to provide monotherapy, adjunctive treatment may not be needed. Moreover, most patients appreciate no longer having to take multiple medications several times a day. The monotherapy and polytherapy approaches were compared indirectly by Lew and colleagues [19] in their assessment of two phase 3 clinical trials. In the first clinical trial, a 54-week, open-label study [8], $92 \%$ of patients were receiving LCIG monotherapy at 4 weeks after the PEG-J procedure [19]. Although $80 \%$ of patients had been on polytherapy at screening and adjunctive therapy was permitted after the initial 4-week treatment period, $76.5 \%$ of patients remained on monotherapy throughout the 54-week study. In contrast, in the second clinical trial, a 12-week, double-blind, placebo-controlled study [6], only oral levodopa was stopped (and replaced by LCIG) while other PD medications were continued at stable doses in $84 \%$ of patients [19]. Efficacy and safety results were comparable whether a monotherapy or polytherapy approach was taken. Moreover, in a subsequent analysis of data from the 54-week study, the LCIG safety profile was similar when used as monotherapy or polytherapy [20]. Notably, the rates and types of adverse events were similar in patients who received LCIG or oral carbidopa/levodopa in the 12-week study [6]. There may be an advantage to a monotherapy approach in that it minimizes the potential for side effects from long-term use of other PD medications. Dopamine agonists, for example, were recently linked to impulse control disorders among patients with PD, the risk for which increased with lifetime average daily dose and duration of treatment [21]. These effects resolved over time after patients discontinued treatment with dopamine agonists. Interestingly, treatment with LCIG has been shown to progressively reduce the symptoms of impulse control disorder, possibly because LCIG provides more stable plasma levodopa levels than does oral levodopa [22, 23].

A table for determining the levodopa equivalent dose of anti-parkinson medications has been previously published by Tomlinson et al. [24]. We have reproduced this table here, with updated information such as dose equivalence for carbidopa/levodopa extended-release capsules (Rytary; Impax Pharmaceuticals, Hayward, CA, USA) [25] and dose-conversion adjustments based on clinical experience (Table 1).

LCIG is provided as single-use, $100-\mathrm{ml}$ cassettes containing $4.63 \mathrm{mg}$ carbidopa and $20 \mathrm{mg}$ levodopa per $\mathrm{ml}$ [26]. LCIG is administered according to three dosing programs: (1) a morning bolus dose, (2) a continuous maintenance dose and (3) extra doses that can be administered as needed. The morning-dose volume is typically calculated by multiplying the patient's standard morning levodopa dose (in $\mathrm{mg}$ ) by 0.8 and dividing the product by $20 \mathrm{mg} / \mathrm{ml}$ (the concentration of LCIG in the cassette). The 0.8 multiplier is used to reduce the risk for dyskinesia; the dose can then be titrated up or down, depending on the sufficiency of the dose. An extra $3 \mathrm{ml}$ is then added to prime the PEG-J tube. An example of the morning-dose calculation is presented in Fig. 3. After the morning-dose administration, which typically takes $10-30 \mathrm{~min}$ depending on the volume being administered, the device automatically transitions to the continuous-dose program.

The continuous infusion rate is determined by subtracting the morning levodopa dose (as calculated above) from the total daytime levodopa daily dose, divided by the number of hours that the infusion was active. The resulting amount in milligrams is then divided by $20 \mathrm{mg} /$ $\mathrm{ml}$ to yield the total LCIG volume that will be administered during the day (except for the morning bolus dose). The hourly infusion rate is calculated by dividing the total LCIG daily volume by the duration of infusion (usually $16 \mathrm{~h}$ ). An example of the continuous-dose rate calculation is shown in Fig. 3.

These morning- and continuous-dose rate calculations provide a starting point from which to make refinements. Suggestions for tailoring LCIG dosing are provided in Table 2. For example, a patient who before transition to LCIG had been receiving a large morning dose of oral levodopa ( $>150 \mathrm{mg}$ ) designed to last 3-4 h may benefit from a smaller LCIG morning bolus dose (e.g., $60 \%$ of the pre-LCIG dose rather than the $80 \%$ used in the standard calculation) because of the continual levodopa 
Table 1 Converting anti-parkinson medications to levodopa equivalent daily dose

\begin{tabular}{ll}
\hline Drug & Conversion factor \\
\hline Immediate-release levodopa & $1 \times$ \\
Controlled-release levodopa & $0 . \times 75^{\mathrm{a}}$ \\
Carbidopa/levodopa extended-release capsules (Rytary) & LD $0.5 \times^{\mathrm{b}}$ \\
Entacapone (or Stalevo) & LD $0.33 \times^{\mathrm{c}}$ \\
Tolcapone & $\mathrm{LD} 0.5 \times^{\mathrm{c}}$ \\
Pramipexole (as salt) & $100 \times$ \\
Ropinirole & $20 \times$ \\
Rotigotine & $30 \times$ \\
Selegiline $10 \mathrm{mg}$ (oral) & $10 \times$ \\
Selegiline $1.25 \mathrm{mg}$ (sublingual) & $80 \times$ \\
Rasagiline & $100 \times$ \\
Amantadine & $1 \times$ \\
Apomorphine & $10 \times$ \\
\hline
\end{tabular}

$L D$ levodopa dose

${ }^{a}$ Based on the authors' clinical experience, a conversion factor of $\times$ 0.6-0.7 may be used for carbidopa/levodopa controlled release (e.g., Sinemet ${ }^{\circledR}$ CR, Merck \& Co. Inc, Whitehouse Station, NJ)

b Based on the conversion from carbidopa/levodopa immediate release to Rytary (Impax Laboratories, Inc., Hayward, CA) published by Pahwa and Lyons [25]

${ }^{c}$ To calculate the total levodopa equivalent daily dose for catechol-O-methyl transferase (COMT) inhibitors (i.e., entacapone, tolcapone), the total levodopa amount (including controlled-release levodopa if COMT inhibitor is given simultaneously) should be calculated then multiplied by the appropriate value. For Stalevo ${ }^{\circledR}$ (Novartis Pharmaceuticals Corp., East Hanover, NJ), the levodopa and COMT inhibitor should be split and calculated separately

Adapted from Tomlinson et al. [24]

infusion with LCIG. Changes in dose will also be guided by patient response and tolerability. The goal of the initial titration visit is to achieve a dose that is neither too high (thus, resulting in excessive dyskinesia, hallucinations/abnormal thinking or behavior) or too low (resulting in "off" time or insufficient improvement in symptoms or objective measures). In cases of significant dyskinesia or dose-responsive side effects, the LCIG dose may need to be reduced. Conversely, if the patient experiences "off" periods, the continuous dose may need to be increased. Similarly, if the morning dose does not reliably induce an "on" state, the dose should be increased. Prescribing guidelines suggest that the morning dose should be increased by $1-2 \mathrm{ml}$ on the subsequent day if the patient demonstrates an inadequate clinical response within $1 \mathrm{~h}$ of morning-dose administration [26]. Often clinicians give patients and caregivers the ability to change the pump setting to increase or decrease the continuous dose by up to $0.5 \mathrm{ml}$ per $\mathrm{h}$, as needed. When clinicians give (appropriate) patients the opportunity to adjust the infusion rate (within a predetermined range), they make the patient essentially a partner in the titration process. In this scenario, the initial titration visit in the clinic can be significantly shortened.

Extra LCIG doses may be self-administered by the patient, as clinically indicated. The LCIG pump allows healthcare providers to set parameters (i.e., volume and time interval between administrations) for these extra doses. 
Example: Patient with a (pre-LCIG) oral morning levodopa dose of $100 \mathrm{mg}$ and a total daily oral levodopa dose of $1300 \mathrm{mg}$

Morning Dose Calculation

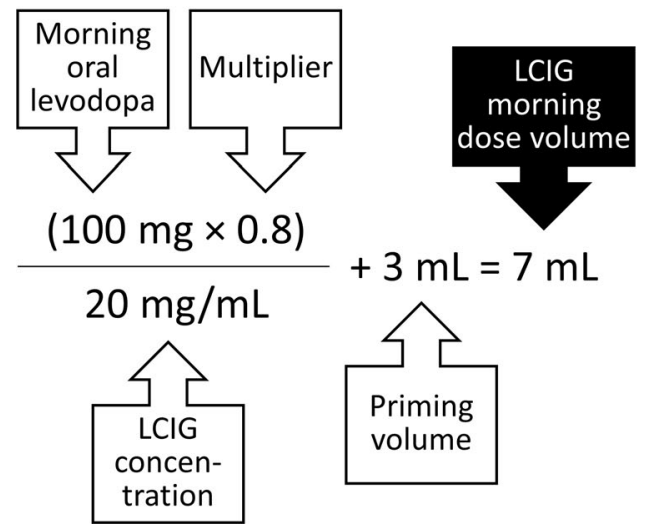

Fig. 3 Calculating levodopa-carbidopa intestinal gel (LCIG) morning bolus dose and continuous infusion rate. Priming volume is the amount of LCIG needed to fill the empty percutaneous endoscopic gastrojejunostomy
Continuous Infusion Rate Calculation

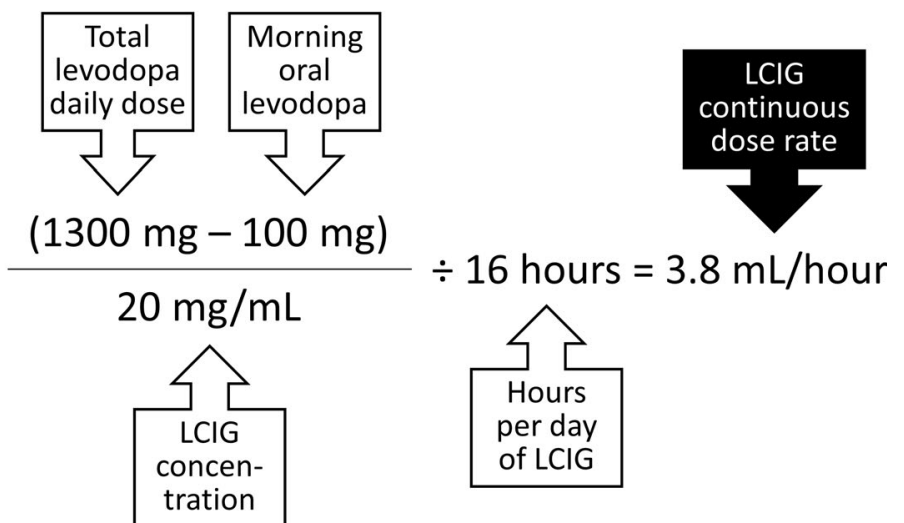

(PEG-J) tube. This is only required for the morning dose calculation, as the tube will be full (or "primed") when the continuous infusion is begun

Table 2 Tips for tailoring levodopa-carbidopa intestinal gel dosing

Decrease the morning dose calculation multiplier from 0.8 to 0.6 for patients who are used to taking large oral levodopa doses $(>150 \mathrm{mg}$ ) with a long interval between the first and second doses

Offer patients the ability to increase or decrease their continuous infusion rate by $0.5 \mathrm{ml} / \mathrm{h}$ to decrease number of visits needed

Offer customized extra-dose settings such as $1 \mathrm{ml}$ every hour (or $2 \mathrm{~h}, 4 \mathrm{~h}$, etc.)

Offer customized extra-dose strengths (e.g., $25 \%$ of hourly infusion rate) rather than the standard $1 \mathrm{ml}$ (equivalent to 20-mg levodopa)

Factor regular use of extra doses into the calculation for maintenance-dose adjustment

The suggested volume of an individual extra dose is $1 \mathrm{ml}$ (20-mg levodopa), but this can be modified at the clinician's discretion. For example, a patient-specific approach may be used, wherein the extra dose is set at $25 \%$ of the hourly infusion rate. Typically, the pump is programmed to require a minimum of $2 \mathrm{~h}$ between extra doses, although some clinicians opt for a smaller between-dose interval (e.g., $1 \mathrm{~h})$. Allowing patients to self-administer extra doses gives clinicians the flexibility to start at a conservative LCIG dose, because patients can use extra-dose "rescue" strategies until the continuous dose can be increased by the clinician. If a patient requires multiple extra doses each day, it suggests that the continuous LCIG dose needs to be increased.

\section{SPECIAL CONSIDERATIONS}

\section{Non-local Patients}

As mentioned above, in the interest of efficiency, patients who must travel a considerable distance to the clinic may prefer to shorten the 
interval between PEG-J tube placement and the initiation of LCIG. Although allowing time for healing is preferred, LCIG treatment can be started within $24 \mathrm{~h}$ of the PEG-J procedure. In Sweden, a pilot program evaluated the feasibility of LCIG titration via remote video communication-based telemonitoring from the patient's home. Remote monitoring was found to be resource-efficient in the setting of nasojejunal LCIG testing prior to permanent PEG-J tube placement, a process that usually requires a multiple-day hospital stay [27]. Although no such remote monitoring has been assessed for patients who proceed directly to PEG-J tube placement, nor has it been studied in the US healthcare system, individual clinicians have reported success with monitoring of patients on LCIG via telephone.

\section{Initiating Titration in the "Off" Versus "On" States}

Physicians have the option of initiating LCIG in the "off" or "on" states. In the former case, the patient would arrive at the clinic on the morning of the first titration visit after missing one levodopa dose (usually his or her morning dose). The clinician would then be able to assess the effectiveness of the morning LCIG dose in the clinic and ensure that the patient feels the effect of the first dose, which normally occurs within $30 \mathrm{~min}$ of administration.

The preference is that the patient arrive in the "off" state to best begin the titration. Patients may come to the clinic having taken their usual oral morning levodopa dose and be in the "on" state on arrival. If the patient is in the "on" state when he or she presents to the clinic, LCIG is initiated at the continuous infusion rate. It is important to note that the PEG-J tube will be empty at the start of the continuous dose in this scenario; therefore, timing of evaluations should include an allowance for pump priming. Using the example in Fig. 3 , at a rate of $3.8 \mathrm{ml} / \mathrm{h}$, priming would take approximately $47 \mathrm{~min}$. If the patient is in the "off" state on arrival at the clinic, an extra dose is given via the LCIG pump and then the continuous infusion is started. Alternatively, some patients arriving in the "on" state start the pump with the morning bolus dose at home, then come to the clinic in the late morning or early afternoon on the continuous infusion for assessment and dose adjustment. The initial continuous rate for these patients is determined by either use of the standard conversion or a custom rate per clinician practice.

\section{Concomitant Use of LCIG with Deep Brain Stimulation}

An increasing body of evidence supports the use of LCIG in patients receiving deep brain stimulation (DBS) who continue to experience refractory symptoms after programming optimization or long-term therapy [28-31]. As the requirement for oral medication increases in these patients, LCIG may offer an advantage over oral levodopa because the fluctuating plasma levels of orally administered levodopa may lead to severe dyskinesia in patients with subthalamic nucleus DBS. DBS settings should be kept stable during the initiation and titration of LCIG, but over time, the relative contribution of DBS and LCIG to the patient's symptom control can be adjusted as needed.

\section{PROGRAMING OPTIONS}

The CADD-Legacy ${ }^{\circledR} 1400$ portable infusion pump (Smiths Medical, Minneapolis, MN, USA) used in the delivery of LCIG has three settings or "lock levels" that govern the degree to which patients can make changes to LCIG dosing. In Lock Level 2, only the healthcare team can make dosing adjustments. This allows the healthcare provider to closely monitor the patient, as any dose modification will require a clinic visit. This type of programming is aligned with the method of care outlined in the pivotal US LCIG study, in which all dosing adjustments were made at the investigator's discretion; patients could not make changes to the infusion rate on their own [6]. In Lock Level 1, the healthcare team can set a dosing range within which patients may make small adjustments (generally $5-10 \%$ or less) to their morning- and continuous-dose rate. The benefit of using this 
option is that patients can immediately and autonomously respond to changes in their motor function, fine-tune their dosing regimen, reduce their dependence on the clinic staff, and gain awareness of their own treatment response. When using Lock Level 1, it is advisable to allow patients to decrease dose and set the upper limit slightly above the anticipated optimal dose. Utilization of Lock Level 1 requires that the patient and/or care partner have the cognitive and motor skills necessary to adjust the pump programming. In our experience, Lock Level 1 is feasible for most patients. Lock Level 0 permits complete access to all programming and operating features and is not typically allowed for patient use.

It is important that any change in the morning- or continuous-dose rate made by the patient, the number of extra doses used, and any need to pause the pump because of excessive dyskinesia are communicated to the clinician so that appropriate adjustments can be made during the next clinic visit. Wearable devices $[32,33]$ or patient diaries may provide additional information on whether the optimal infusion rate has been achieved and whether the goal of minimal "off" time and maximal "on" time without bothersome dyskinesia has been reached when the patient is in his or her home environment.

\section{PATIENT EDUCATION AND TRAINING}

The process of patient education and training begins with the discussion of LCIG as a potential treatment option, as either an immediate consideration or a future possibility. When feasible, the patient's care partner should be involved in these discussions, particularly if he or she will be called on to assist in the setup and management of the system and to provide reliable information regarding symptoms (e.g., "on" and "off" time). The amount and type of information relayed should align with the patient's stage in the process. For example, for a patient who has opted to initiate LCIG and is scheduled for PEG-J tube placement, a discussion with the patient and care partner should provide procedural information and identify the individuals involved in the care team and their responsibilities. This information will help direct the patient or care partner to the appropriate contact should he or she have questions or experience issues. Concerns regarding the PEG-J tube or wound site are best managed by the proceduralist, whereas other questions should be directed to the neurologist and treatment team or, as appropriate, DuoConnect, the manufacturer-sponsored patient and healthcare provider support program. DuoConnect also provides nursing support via phone and home health visits (if desired), which typically occurs before PEG-J tube placement, $24 \mathrm{~h}$ after the procedure, and on the day following LCIG initiation. This nursing education and support is a valuable resource during the titration process, particularly for patients who initiate LCIG therapy for the first time at home with the morning bolus dose.

Instruction regarding the proper operation of the pump device is provided by the clinic staff during the initial titration visit. If possible, introduction to a mock device prior to the initial titration visit can help familiarize patients with the pump. It is imperative that patients and their care partners demonstrate their understanding of the equipment and be able to operate the pump prior to leaving the clinic on the first titration visit.

\section{SUBSEQUENT TITRATION AND FOLLOW-UP}

After the initial titration visit, the first follow-up visit can be as early as the following day, but usually occurs after 2-6 weeks. For patients who have been given the ability to make dose adjustments within a prespecified range (i.e., Lock Level 1), the interval between the first and second visit may be extended. The goal of a clinic visit conducted the morning after the initial titration is to ensure that the patient is using the pump properly and to make any necessary dose adjustments. Changes in dosing at this point are generally minor $(<10 \%)$. Patients will arrive at the clinic in the "on" state 
and will be at the facility from as little as $30 \mathrm{~min}$ to a maximum of approximately $2 \mathrm{~h}$.

During subsequent clinic visits, progressively less time will be spent discussing device or dosing issues and visits will revert back to regular office visits. The only major change is that the medication regimen has become much simpler, with LCIG monotherapy being the rule rather than the exception. The number of extra doses being used on a regular basis should be taken into consideration. If there is a regular/daily pattern in extra dose usage (e.g., $\geq 5$ extra doses every day), increasing the continuous infusion rate is warranted. If, however, a patient uses multiple extra doses on occasion for difficult days, the continuous dose may not need to be adjusted, but consideration should be given to broadening the range on the Lock Level 1 programming. Increasing the Lock Level 1 dosing range is also advisable if concomitant anti-parkinson medications are being reduced or phased out. Much like oral levodopa treatment, in our clinical experience, decisions regarding dosing and dose changes should also factor in tolerability, with clinicians querying patients or performing relevant assessments related to known carbidopa/levodopa side effects, such as somnolence, orthostatic hypotension, signs of an impulse control disorder, depression, suicidal ideation, neuropathy, symptoms of ischemic heart disease or arrhythmia, melanoma, and glaucoma [26].

\section{ACHIEVING AN OPTIMAL LCIG DOSE}

Over time, adjustments in LCIG dose will be warranted, as they would be with any antiparkinson medication. During the short term, reaching an optimal dose can be defined as the point at which dose adjustment becomes less frequent. In clinical trials, where patient assessment timing and titration methodology were standardized, a stable LCIG dose was achieved by most patients in approximately 1 week $[6,10]$. In clinical practice, once the patient feels his or her motor fluctuations are adequately controlled and the practitioner sees sufficient improvement on objective and subjective measures, further dosing adjustments can be made as part of routine clinic visits (generally every 3-6 months). If a previously effective infusion rate is no longer effective, the clinician may need to investigate to determine if the tube has moved from its initial placement.

\section{CONCLUSIONS}

Many approaches to provide continuous, nonpulsatile dopaminergic stimulation are being pursued, including gastrointestinal pump-based gels containing catechol-O-methyl transferase inhibitors, levodopa pumps attached to a transdermal patch, and subcutaneous pumpbased dopamine agonists [32]. The approval of LCIG has added another treatment option to the limited armamentarium for patients with advanced PD. Utilization of LCIG is growing in the US as clinicians gain familiarity with its use, but LCIG treatment still lags behind treatment provided in other countries where LCIG has been available for more than 10 years. Perceptions of titration complexity may inhibit clinicians from pursuing LCIG therapy for appropriate patients. However, emerging clinical data and experience with LCIG outside the clinical trial setting have shown that titration can be easily managed on an outpatient basis. Moreover, flexibility in scheduling and individualized therapeutic regimens allow clinicians to tailor the titration process to the patient and to their practices.

\section{ACKNOWLEDGEMENTS}

Funding. Development of this manuscript, including the Rapid Service Fee, was sponsored by AbbVie, Inc., North Chicago, IL, USA. AbbVie participated in the interpretation of data, writing, reviewing, and approving the manuscript for publication.

Medical Writing and Other Editorial Assistance. Crystal Murcia, PhD, and Kelly $\mathrm{M}$. Cameron, PhD, ISMPP CMPP, of JB Ashtin provided medical writing and editorial support to 
the authors in the development of this manuscript; medical writing support was funded by AbbVie.

Authorship. All named authors meet the International Committee of Medical Journal Editors (ICMJE) criteria for authorship for this article, take responsibility for the integrity of the work as a whole, and have given their approval for this version to be published. All authors had full access to the articles reviewed in this manuscript.

Disclosures. Fahd Amjad has received research support/honoraria from AbbVie, Teva Pharmaceutical Industries, UCB, Adamas Pharmaceuticals, US WorldMeds, Merz, and Lundbeck for consulting or serving on advisory boards. Danish Bhatti has performed speaking/consulting activities for ACADIA Pharmaceuticals, Teva Pharmaceutical Industries, AbbVie, Merz, and Adamas Pharmaceuticals. Thomas L. Davis has received research support/ honoraria from AbbVie for consulting or serving on advisory boards. Odinachi Oguh has received research support/honoraria from AbbVie and Adamas Pharmaceuticals for consulting or serving on advisory boards. Rajesh Pahwa has received consulting fees from AbbVie, ACADIA Pharmaceuticals, Acorda Therapeutics, Adamas Pharmaceuticals, Cynapsus Therapeutics, Global Kinetics, Ionis Pharmaceuticals, Lundbeck, Neurocrine Biosciences, St. Jude Medical, Teva Neuroscience, UCB, and US WorldMeds. He has received research grants from Acorda Therapeutics, Adamas Pharmaceuticals, Avid Radiopharmaceuticals, Boston Scientific, Cala Health, Cynapsus Therapeutics, Kyowa, National Parkinson Foundation, NIH/NINDS, Parkinson Study Group, Pfizer, and US WorldMeds. Leonard V. Metman has received research support/ honoraria from Abbott, AbbVie, Boston Scientific, and Medtronic for consulting or serving on advisory boards. He has received research support from AbbVie, Adamas Pharmaceuticals, Boston Scientific, Medtronic, Neuroderm Ltd, NIH, Pfizer, and US WorldMeds. Pavnit Kukreja is an employee of AbbVie. Inc., and may own stock or stock options. Jorge Zamudio is an employee of AbbVie. Inc., and may own stock or stock options.

Compliance with Ethics Guidelines. This article is based on previously conducted studies and the authors' clinical experience and does not involve any new studies of human or animal subjects performed by any of the authors.

Data Availability. Data sharing is not applicable to this article as no datasets were generated or analyzed during the current study.

Open Access. This article is distributed under the terms of the Creative Commons Attribution-NonCommercial 4.0 International License (http://creativecommons.org/licenses/ by-nc/4.0/), which permits any noncommercial use, distribution, and reproduction in any medium, provided you give appropriate credit to the original author(s) and the source, provide a link to the Creative Commons license, and indicate if changes were made.

\section{REFERENCES}

1. Epstein M, Johnson DA, Hawes R, Schmulewitz N, Vanagunas AD, Gossen ER, et al. Long-term PEG-J tube safety in patients with advanced Parkinson's disease. Clin Transl Gastroenterol. 2016;7:e159.

2. Othman AA, Rosebraugh M, Chatamra K, Locke C, Dutta S. Levodopa-carbidopa intestinal gel pharmacokinetics: lower variability than oral levodopa-carbidopa. J Parkinsons Dis. 2017;7(2):275-8.

3. Verghagen Metman L, Konitsiotis S, Chase TN. Pathophysiology of motor response complications in Parkinson's disease: hypotheses on the why, where, and what. Mov Disord. 2000;15(1):3-8.

4. Kurth MC, Tetrud JW, Tanner CM, Irwin I, Stebbins GT, Goetz CG, et al. Double-blind, placebo-controlled, crossover study of duodenal infusion of levodopa/carbidopa in Parkinson's disease patients with 'on-off' fluctuations. Neurology. 1993;43(9):1698-703.

5. Nyholm D, Nilsson Remahl AI, Dizdar N, Constantinescu R, Holmberg B, Jansson R, et al. Duodenal levodopa infusion monotherapy vs oral polypharmacy in advanced Parkinson disease. Neurology. 2005;64(2):216-23. 
6. Olanow CW, Kieburtz K, Odin P, Espay AJ, Standaert DG, Fernandez HH, et al. Continuous intrajejunal infusion of levodopa-carbidopa intestinal gel for patients with advanced Parkinson's disease: a randomised, controlled, double-blind, doubledummy study. Lancet Neurol. 2014;13(2):141-9.

7. Antonini A, Yegin A, Preda C, Bergmann L, Poewe W, Gloria Study Investigators. Global long-term study on motor and non-motor symptoms and safety of levodopa-carbidopa intestinal gel in routine care of advanced Parkinson's disease patients; 12-month interim outcomes. Parkinsonism Relat Disord. 2015;21(3):231-5.

8. Fernandez $\mathrm{HH}$, Standaert DG, Hauser RA, Lang AE, Fung VS, Klostermann F, et al. Levodopa-carbidopa intestinal gel in advanced Parkinson's disease: final 12-month, open-label results. Mov Disord. 2015;30(4):500-9.

9. Slevin JT, Fernandez HH, Zadikoff C, Hall C, Eaton $\mathrm{S}$, Dubow J, et al. Long-term safety and maintenance of efficacy of levodopa-carbidopa intestinal gel: an open-label extension of the double-blind pivotal study in advanced Parkinson's disease patients. J Parkinsons Dis. 2015;5(1):165-74.

10. Standaert DG, Rodriguez RL, Slevin JT, Lobatz M, Eaton S, Chatamra K, et al. Effect of levodopa-carbidopa intestinal gel on non-motor symptoms in patients with advanced Parkinson's disease. Mov Disord Clin Pract. 2017;4(6):829-37.

11. Antonini A, Poewe W, Chaudhuri KR, Jech R, Pickut B, Pirtosek Z, et al. Levodopa-carbidopa intestinal gel in advanced Parkinson's: final results of the GLORIA registry. Parkinsonism Relat Disord. 2017;45:13-20.

12. Kruger R, Lingor $P$, Doskas $T$, Henselmans JML, Danielsen EH, de Fabregues $\mathrm{O}$, et al. An observational study of the effect of levodopa-carbidopa intestinal gel on activities of daily living and quality of life in advanced Parkinson's disease patients. Adv Ther. 2017;34(7):1741-52.

13. De Fabregues O, Dot J, Abu-Suboh M, HernandezVara J, Ferre A, Romero O, et al. Long-term safety and effectiveness of levodopa-carbidopa intestinal gel infusion. Brain Behav. 2017;7(8):e00758.

14. Odin P, Ray Chaudhuri K, Slevin JT, Volkmann J, Dietrichs E, Martinez-Martin P, et al. Collective physician perspectives on non-oral medication approaches for the management of clinically relevant unresolved issues in Parkinson's disease: consensus from an international survey and discussion program. Parkinsonism Relat Disord. 2015;21(10):1133-44.

15. Dowell P, Lyons K, Pahwa R. Clinical experience with initiation of carbidopa/levodopa enteral suspension in Parkinson's disease patients with cognitive impairment. Mov Disord. 2017;32(Suppl 2):S287.

16. Fasano A, Liu LW, Poon YY, Lang AE. Initiating intrajejunal infusion of levodopa/carbidopa intestinal gel: an outpatient model. Mov Disord. 2015;30(4):598-9.

17. Aldred J, Davis T, Zamudio J, Kukreja P, Bergmann $\mathrm{L}$, Li M, et al. Titration of levodopa-carbidopa intestinal gel in US patients with advanced Parkinson's disease. Mov Disord. 2018;33(Suppl 1):S93.

18. Solla P, Fasano A, Cannas A, Mulas CS, Marrosu MG, Lang AE, et al. Dopamine agonist withdrawal syndrome (DAWS) symptoms in Parkinson's disease patients treated with levodopa-carbidopa intestinal gel infusion. Parkinsonism Relat Disord. 2015;21(8):968-71.

19. Lew MF, Slevin JT, Kruger R, Martinez Castrillo JC, Chatamra K, Dubow JS, et al. Initiation and dose optimization for levodopa-carbidopa intestinal gel: insights from phase 3 clinical trials. Parkinsonism Relat Disord. 2015;21(7):742-8.

20. Boyd JT, Zadikoff C, Benesh JA, Zamudio J, Robieson WZ, Kukreja P. Safety and efficacy of levodopa-carbidopa monotherapy in patients with advanced Parkinson's disease. J Neuro Sci. 2017;381(Suppl):182-3.

21. Corvol JC, Artaud F, Cormier-Dequaire F, Rascol O, Durif F, Derkinderen P, et al. Longitudinal analysis of impulse control disorders in Parkinson disease. Neurology. 2018;91(3):e189-201.

22. Catalan MJ, Molina-Arjona JA, Mir P, Cubo E, Arbelo JM, Martinez-Martin P, et al. Improvement of impulse control disorders associated with levodopa-carbidopa intestinal gel treatment in advanced Parkinson's disease. J Neurol. 2018;265(6):1279-87.

23. Evans AH, Pavese N, Lawrence AD, Tai YF, Appel S, Doder $\mathrm{M}$, et al. Compulsive drug use linked to sensitized ventral striatal dopamine transmission. Ann Neurol. 2006;59(5):852-8.

24. Tomlinson CL, Stowe R, Patel S, Rick C, Gray R, Clarke CE. Systematic review of levodopa dose equivalency reporting in Parkinson's disease. Mov Disord. 2010;25(15):2649-53.

25. Pahwa R, Lyons KE. Outpatient titration of carbidopa/levodopa enteral suspension (Duopa). Int J Neurosci. 2017;127(5):459-65.

26. Duopa (carbidopa and levodopa) enteral suspension [prescribing information]. North Chicago: AbbVie Inc. 2016. https://www.rxabbvie.com/pdf/ duopa_pi.pdf. 
27. Willows T, Dizdar N, Nyholm D, Widner H, Grenholm P, Schmiauke $\mathrm{U}$, et al. Initiation of levodopa-carbidopa intestinal gel infusion using telemedicine (video communication system) facilitates efficient and well-accepted home titration in patients with advanced Parkinson's disease. J Parkinsons Dis. 2017;7(4):719-28.

28. Klostermann F, Jugel C, Marzinzik F. Jejunal levodopa infusion in long-term DBS patients with Parkinson's disease. Mov Disord. 2011;26(12): 2298-9.

29. Burack MA, Santiago A, Biglan K, Barbano R. Levodopa-carbidopa intestinal gel therapy (LCIG) choices and clinical outcomes in DBS-eligible versus palliative stage PD patients. Parkinson Study Group Annual Meeting; September 17-19; Portland, Oregon; 2016.
30. Kumar N, Murgai A, Naranian T, Jog M, Fasano A. Levodopa-carbidopa intestinal gel therapy after deep brain stimulation. Mov Disord. 2018;33(2):334-5.

31. Regidor I, Benita V, de Pedro MD, Ley L, Martinez Castrillo JC. Duodenal levodopa infusion for longterm deep brain stimulation-refractory symptoms in advanced Parkinson disease. Clin Neuropharmacol. 2017;40(3):103-7.

32. Blaze RL, Tan J, Evans AH. Quantitative assessment of advanced therapies in Parkinson's disease using the Parkinson kinetigraph (PKG). Mov Disord. 2016;31(Suppl 2):S189.

33. Margolesky J, Luca C. Personal KinetiGraph devices assessing efficacy of continuous enteral carbidopa/ levodopa infusion therapy. Mov Disord. 2017;32(Suppl 2):S267. 